\title{
A Fuzzy Approach For The 2007 CIG Simulated Car Racing Competition
}

\author{
Duc Thang Ho and Jonathan M. Garibaldi
}

\begin{abstract}
This paper describes the techniques that have been used by the winning entry of the 2007 IEEE Congress on Evolutionary Computation (CEC2007) and the CIG2007 car racing competitions. The challenge is to race against an opponent around a track, trying to get as many points as possible. Previous research on similar problems are mostly based on either state-based or action-based controller architectures trained with machine learning techniques. In this paper, a hybrid controller architecture is presented, combining both the advantages of the existing architectures. The main component of the controller is designed as fuzzy systems whose membership functions are changeable according to the context. Finally, the competition results are given.
\end{abstract}

\section{INTRODUCTION}

Car racing is a challenging problem, attracting public excitement, which is evident from huge amount of money invest in both practicing and watching physical car racing. Developing a good car racing controller is also very challenging which requires knowledge of the car's behaviour in different environments and various forms of real-time planning, such as path planning and overtaking planning, etc. Computational intelligence techniques have been applied to physical car racing such as in the DARPRA Grand Challenge [1] or in the radio-controller toy car racing which were run as a competition for IEEE Congress on Evolutionary Computation (CEC) for 2003, 2004 and 2005. These challenges have yet to see a controller that is good enough to be considered competitive with a competent human racer.

On the other hand, simulations of the racing challenge are also interesting and challenging in their own right. Togelius and Lucas [2][3][4] investigated various controller architectures and sensor input representations for simulated car racing. It was found that controllers based on first-person sensory inputs and neural networks could be evolved to achieve a better performance than humans over a number of racetracks, and display interesting behaviours when coevolved with another car on the same track [5]. Most often various learning methods for developing controllers for car racing simulations or games are used by the researchers. Floreano et al. [6] developed a first-person controller using a small part $(5 \times 5$ pixels $)$ of the visual field as the input to a neural network. The evolved controller successfully drove the car around the track. Chaperot [7] applied advanced artificial neural network trained with Evolutionary Algorithms and Back-propagation Algorithm in a motocross game with the results claimed to be better than any living player. Abbeel

D. T. Ho and J. M. Garibaldi, School of Computer Science, University of Nottingham, Nottingham, UK NG8 1BB ; Email: dthecs.nott.ac.uk and jmg@cs.nott.ac.uk and $\mathrm{Ng}$ [8] trained a system using reinforcement learning in a flight simulator and driving a real radio-controlled car. Coulom [9] in his $\mathrm{PhD}$ thesis, presented an efficient pathoptimization algorithm that was used to train his K1999 car driver of the Robot Auto-Racing Simulator (RARS) [10]. K1999 won the 2000 and 2001 RARS formula one seasons.

\section{A. Control architecture}

After a careful examination of existing control architectures, it appears that the state-based and action-based architectures are the two most frequently used. The classical state-based approach consists in computing an optimal trajectory first. Then, a controller was built to track this trajectory. Examples of successful controllers of this type include the $K 1999$ controllers of Coulom [9] in the RARS simulation, the berniw3 controller of Wymann [11] in the The Open Racing Car Simulator (TORCS) [12], etc. This type of approach favors high-level reasoning capabilities, i.e planning and often achieves excellent performance in the case of the track environment is completely known and fixed. The action-based controllers, on the other hand, favors reactivity as actions follow perceptions closely, almost like a reflex. Examples of successful controllers include the non-stationary fuzzy controller that won the FuzzIEEE 2007 car racing competition by Ho\&Garibaldi [13], various machine learning controllers developed by Togelius and Lucas [2][3][4], etc. In general, the state-based approaches often outperform the action-based approaches if both can be applied. However, there are some limitations which make the state-based architecture unable to deal with dynamic and uncertain environments in real-time. First, computing an optimal trajectory is often too costly to be perform online. Second, it cannot be assumed that the environment will stay the same as when the planning takes place. So, whenever an unexpected event happens, it is required to perform the expensive planning process again. And finally, the planning can only be applied when a forward model exists, which is able to predict the next state of the car given the current state and the selected actions. If such model is not directly available, it must be learned first. In this paper, a hybrid control architecture which combines both the high-level reasoning capabilities of the state-based approach and the reactivity of the action-based approach is presented. The key component of the control architecture that was used to control the car, is designed as a fuzzy controller, i.e. a controller based upon fuzzy logic, thus permitting approximate reasoning and a human-like description of the car's reactive behaviours. This fuzzy component differs from 
classical fuzzy approaches in that the membership functions which were used to construct the fuzzy sets of the controller are changeable in accordance to the context. Both the control architecture and the its components will be detailed in section 3. Section 2 describes the characteristics of the car racing simulation we will be using. Experimental results are given in section 4. And finally, conclusion are drawn and further research is suggested in section 5 .

\section{THE CAR RACING MODEL}

The racing game model in this paper is the same as that was used for the simulated car racing competition at the 2007 IEEE Computational Intelligence and Games Symposium and the 2007 IEEE Congress on Evolutionary Computation. In this game, one or two cars race in an arena without walls. The objective of the game is to reach as many way points as possible within 500 time steps. These way points appear within a $400 \times 400$ pixel square area; the cars are not bounded by this area and can drive as far from the center of the arena as they want. Way points are randomly positioned within a circular radius at the start of each trial, so that no two trials are identical; at any point two way points are visible to human or autonomous controllers, but only one way point (the current way point) can be "taken" by a car at any occasion. As soon as the current way point is reached, the way point counter is incremented for that car, the other visible way point (the next way point) becomes the current, and a new next way point is generated at a random position. See Fig. 1 for a depiction of the game.

At any time step, the car controller is given a model of the sensors which include the current states of the car and its opponent, i.e velocity, orientation, position, etc., as well as the positions of the visible way points. The controller is then required to return one of the following actions: back, back-right, back-left, neutral, left, right, forward, forward-left and forward-right. The dynamics of the car are reasonably realistic, so acceleration and deceleration take time, and turning while traveling at high speed will cause considerable skidding. Turning on the spot is certainly not possible. Carto-car collisions are possible and result in both Victoria and angular impetus.

Despite its apparent simplicity in terms of rules, this game has plenty of hidden complexity that a controller needs to solve.

- Reach the current way point as fast as possible without overshooting it.

- Reach the current way point in such a way that the next way point can be reached quickly.

- Predict which car will reach the current way point first and take appropriate actions.

- Handle collisions effectively.

- Predict opponent's behaviours and take appropriate actions.

More details about the competitions can be gathered and its complete source code downloaded from the organiser's website at http://julian.togelius.com/cig2007competition and

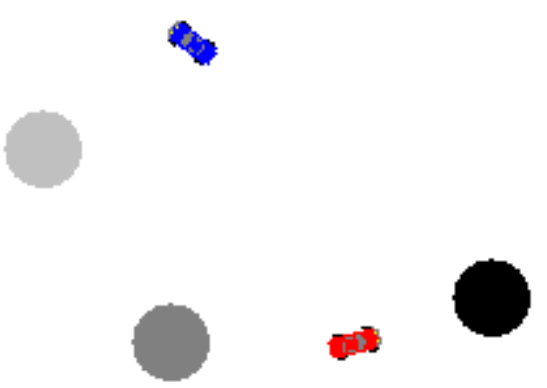

Fig. 1. Two cars in the point-to- point racing game. The black circle represents the current way point, the dark gray circle the next way point, and the light gray circle the next way point after that

http://julian.togelius.com/cec2007competition. Interested readers could refer to the paper of Togelius and others [14] for more in-depth details of the rules and the physical model used in this game.

\section{CONTROL ARCHitecture}

On the top layer, the control architecture consists of two main components:

- Waypoint chooser: uses a simple internal heuristic controller to estimate the number of steps required for each car to hit the first waypoint. If our car takes fewer steps to get to the first waypoint than the opponent car, then the first is selected as the target and the second waypoint is the next target, otherwise the second waypoint is selected as both the target and the next target because there are only two way points visible at a time.

- The main controller: takes the current states of the car and the output targets of the waypoint chooser to calculate the final action. In the case of solo race, the waypoint chooser will always output the first way point visible as the current target that the car should drive to and the second visible way point as the next target that the car should prepare for before visit the current target. The aim of the main controller is to drive the car to the target so that when the car hits the target, it has a heading angle generally towards the next target.

The focus of this paper is on the main controller component only and the reader is referred to Figure 2 for a complete presentation of the architecture.

\section{A. The main controller}

The following scenarios are implemented:

- The target and the next target are the same: the car slows down on the approach and stops exactly at the target way point. The car will wait for the first waypoint to be taken by the opponent in which case the second waypoint becomes active and so will be taken immediately. To resolve the potential problem when the opponent car runs into an infinite circular loop trying to get the first waypoint while our car is waiting at the second waypoint, we allow our car to wait for maximum of 


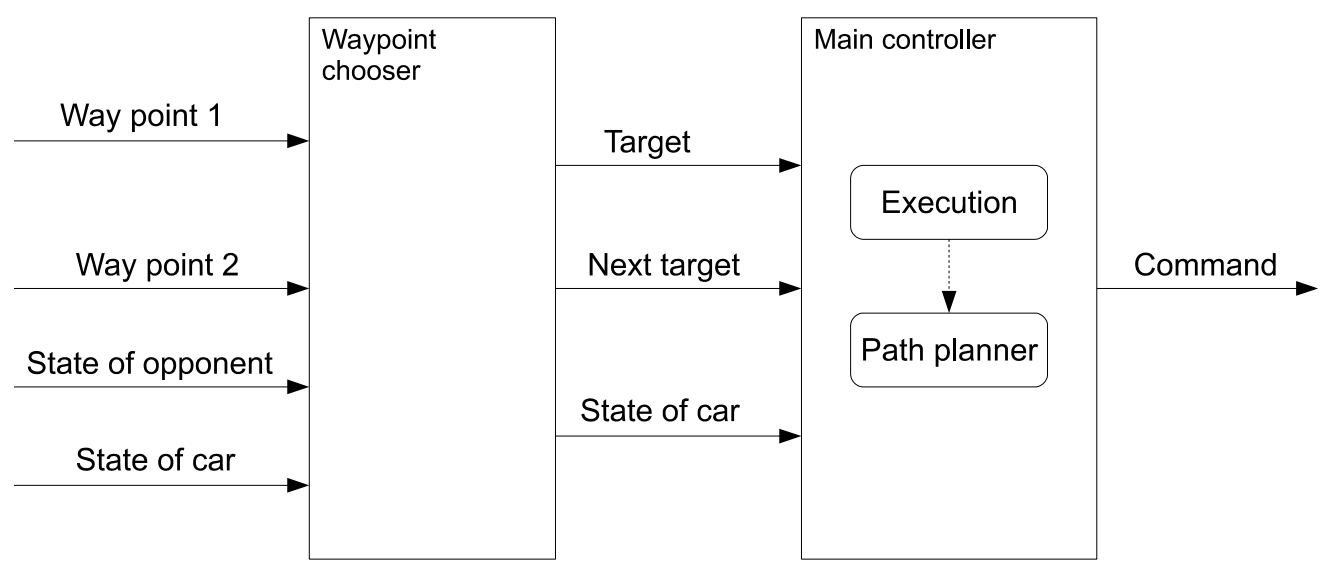

Fig. 2. The control architecture of the racing car

100 steps at the second waypoint. If the waiting time is elapsed and the first waypoint is not taken, the car stops waiting and changes the target to the first waypoint.

- The target and the next target are different : The aim is to drive the car to the target way point so that when the car hits the target, it has a heading angle generally towards the next target.

In this paper, only the second scenario is detailed. From now, it is assumed that the target and next target way points are different.

\section{B. Control architecture of the main controller}

The main controller consists of two main modules that are complementary: the path planner and the execution controller. In classical state-based approach, the path planner is used to compute an optimal path and the execution controller pilots the car to follow that path as closely as possible. In our approach, we aim to combine both high-level reasoning capabilities and reactivities (so as to be able to deal with unexpected events in due time). Therefore, we decided to implement the execution controller as the key component of the system with the capability of a stand-alone action-based controller without referring to any pre-computed path. We opt for a fuzzy approach when implementing the execution controller as we hope to endow the controller with the human-like reactive capabilities required in an uncertain and partially known environment like the point-to-point racing game. The path planner module uses the execution controller to pre-compute different trajectories and returns the best option. This is a very expensive operation and thus is only used when the situation is simplified enough. The purpose of the path planner is to incorporate the power of machine computations to enhance the performance of the human-like solution given by the execution controller. Both of these modules will be detailed in the following sub-sections.

\section{Execution controller}

As mentioned earlier, the goal of the execution controller is to generate commands for the car so as to react to situations in real-time. The execution controller is designed as two fuzzy controllers, the speed controller and the steering controller, to control the acceleration and the steering of the car respectively. These two controllers take into account the positions of the two target way-points and the current state of the car in order to produce the desired speed and desired steering that the car should achieve at the next step. The outputs of the controllers are then combined into a single command which minimizes the difference between the current state of the car and the desired state. Before detailing execution controller and its main features, let us briefly recall what a fuzzy controller is.

1) Fuzzy controller: Fuzzy controller is a control system based on the Zadeh's theory of fuzzy sets [15], thus permitting approximate reasoning and a human-like description of system's behaviours. A typical fuzzy controller consists of four components:

- The knowledge base: encodes the desired behaviours of the process. It is made up of:

- Fuzzy sets: sets of membership functions associated with each input and output variable of the system.

- Fuzzy rules: human-like rules of the form "IF condition THEN action".

- The fuzzifier: turns real input values into fuzzy values, i.e set of (fuzzy set label, membership degree) couples. 

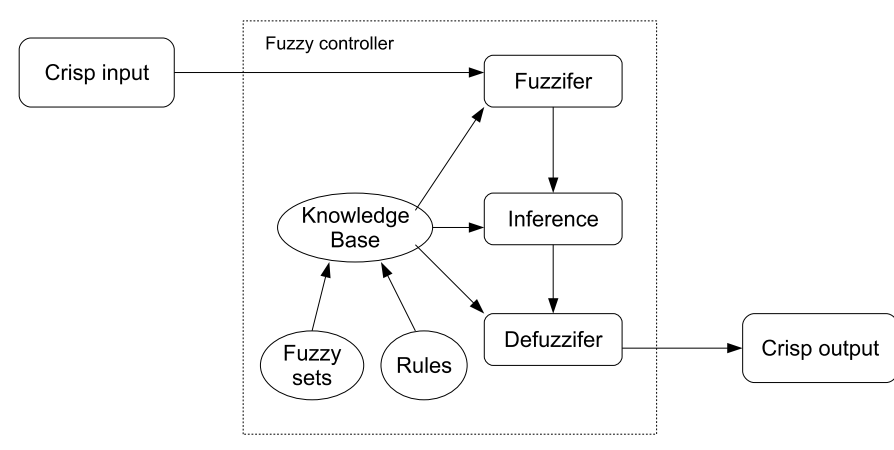

Fig. 3. The architecture of a fuzzy controller

- The inference engine: is the kernel of the fuzzy controller. It performs fuzzy inference from the input fuzzy sets based on the rules of the system and output fuzzy sets

- The defuzzifier: turns an output fuzzy set to an output real value.

Figure 3 shows the architecture of a typical fuzzy controller. The interested reader is particularly referred to [16] for a summary of tutorial and for more details.

2) Main features of execution controller: The speed and steering controllers of the execution controller are designed as fuzzy controllers, as such, each of them includes four components as described above. However, they differ from the classical fuzzy controllers in that the membership functions of the fuzzy sets are not fixed but dependent on a set of linguistic variables called the context set. Formally, let us consider an fuzzy controller system which consists of $n$ linguistic variables. Each linguistic variable $x_{i}, i \in\{1, \ldots, n\}$, has a universe of discourse $X_{i}$. The context set $C$ is defined to be a subset of the Cartesian product of the universe of discourse $X_{1}, \ldots, X_{n}$ :

$$
\mathcal{C} \subseteq X_{1} \times \ldots \times X_{n}
$$

For each context $c \in \mathcal{C}$, the context-dependent fuzzy set of a term $\bar{A}$ of a linguistic variable $x_{i}$ is defined as:

$$
\bar{A}=\int_{c \in \mathcal{C}} \int_{x_{i} \in X_{i}} \mu_{\bar{A}}\left(c, x_{i}\right) / x_{i} / c .
$$

Note that the variables in the context set do not necessarily appear in the rule set of the fuzzy system. The linguistic variables of a context sets could be the input variables, the output variables or any other variables that the system has the knowledge of. For example, we could have a context set consisting of three variables, distance, speed and heading angle. However, only speed and heading angle are used in the rule set of the system and distance is only used as a dummy variable which influences the fuzzy sets of the other two variables. The context set $\mathcal{C}$ can contain any number of variables in the fuzzy system. Each specific instant $c$ of the context set $C$ consists of the states of the variables defined in $C: c=\left\{x_{1}, \ldots, x_{k}\right\}$. It is implicitly assumed that the variable

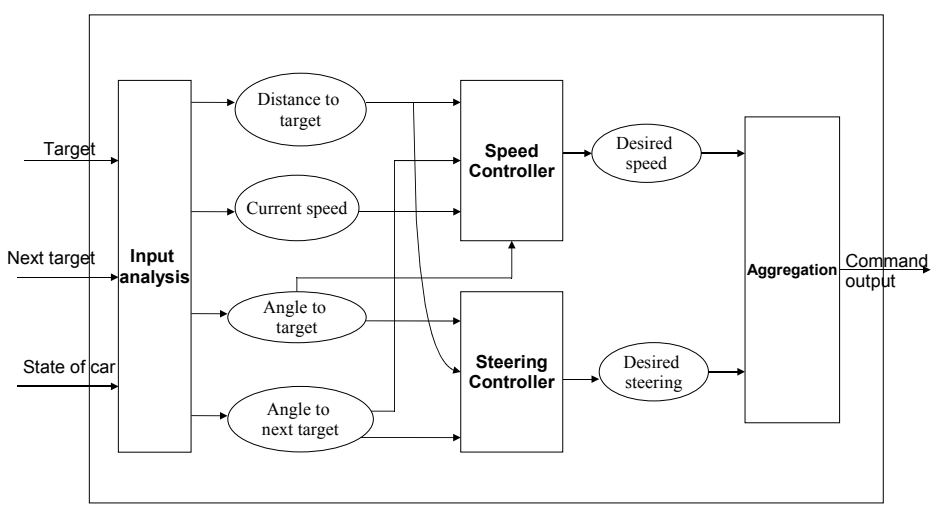

Fig. 4. Design of the execution controller

$x_{i}$ of the context will not have any effect on the variable $x_{i}$ of the system to avoid recursive definitions. Fuzzy sets defined based on context sets are called context-dependent fuzzy sets. It is hoped that with context-dependent fuzzy sets, it is able to model the effect of the context environments on the inference process. A fuzzy controller which uses at least one contextdependent fuzzy set is a context-dependent fuzzy controller. The interested reader is particularly referred to [13] for more details. See Figure 4 for an overview of the design structure of the execution controller.

3) Linguistic variables and fuzzy sets: There are six linguistic variables: distance, speed, heading angle, next angle, desired speed and desired steering. The first four variables are inputs to the fuzzy controllers and the final two are the outputs.

Five fuzzy sets labeled VeryNear, Near, Medium, Far and VeryFar are associated with the distance variable. Similarly, nine fuzzy sets la belled BehindLeft, BehindRight, HardLeft, HardRight, LeftDir, RightDir, SmallLeft, SmallRight and StraightAhead are associated with the current heading angle variable and the next angle variable. The speed variable of the input and the desired speed variable of the output associated with seven fuzzy sets: VerySlow, Slow, Medium, Fast, VeryFast, BFast and Backward. There are only three crisp sets associated with the desired steering output: 1 for Left, 0 for Neutral and -1 for Right. Figure 5 depicts the fuzzy sets associated with the distance variable. Figure 6 depicts the fuzzy sets associated with the heading angle and the next angle variables. The fuzzy sets of the speed variable and the desired speed variable are shown in Figure 7. The desired steering output are drawn in Figure 8.

All the fuzzy sets are chosen empirically and at no time has any training or parameter tuning taken place in the implementation of the controller. All the linguistic variables with their fuzzy sets used in the speed controller are exactly as shown in the figures. In the steering controller, we want to have the fuzzy sets labeled LeftDir, RightDir, SmallLeft, SmallRight and StraightAhead of the current heading angle variable depending on the distance variable, thus make them become context-dependent fuzzy sets. We define $\theta$ as a 


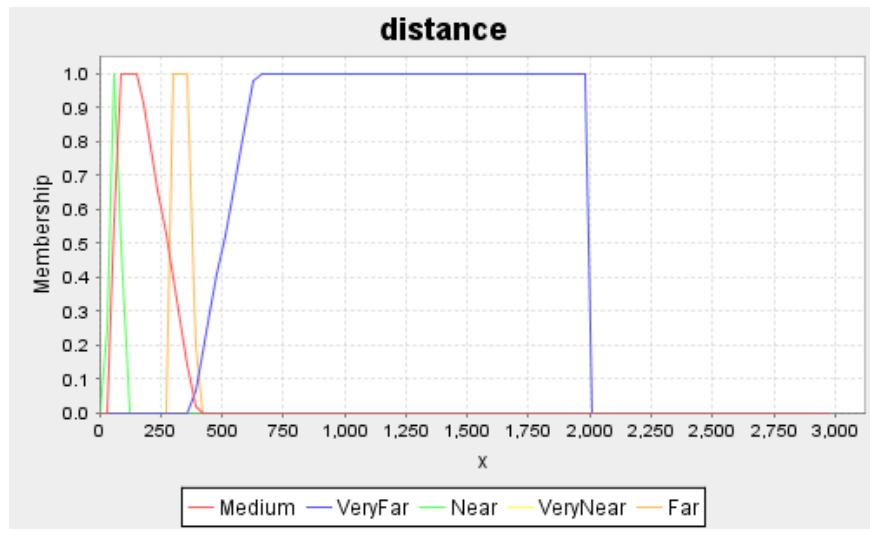

Fig. 5. Five fuzzy sets associated with the distance variable

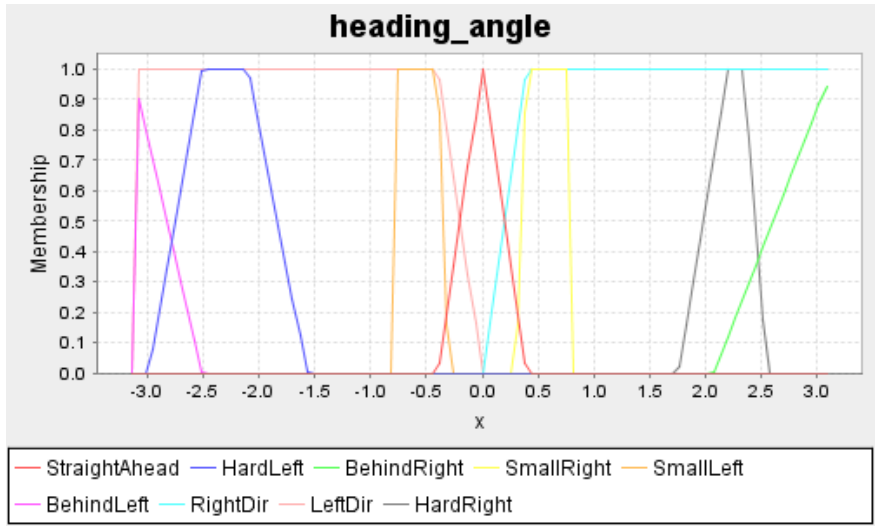

Fig. 6. The nine fuzzy sets associated with the heading angle and next angle variables

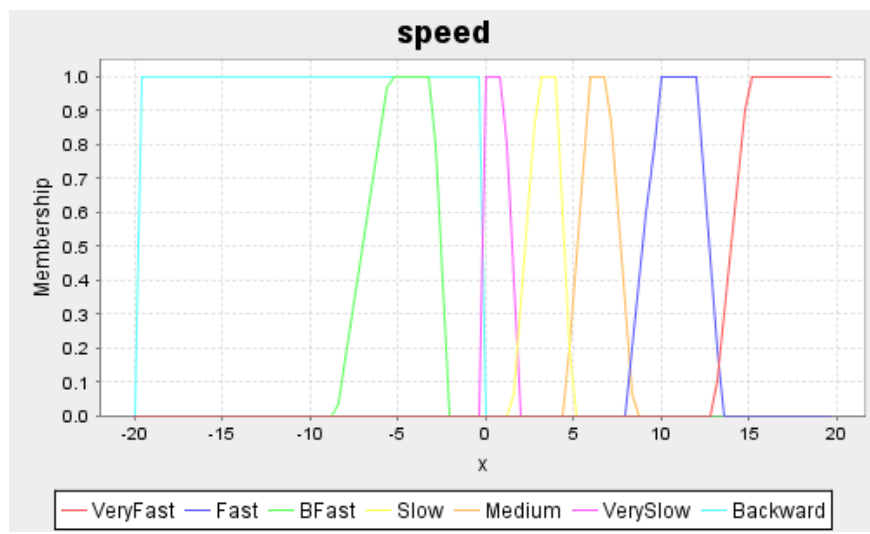

Fig. 7. Seven fuzzy sets associated with the input variable speed and output desired speed

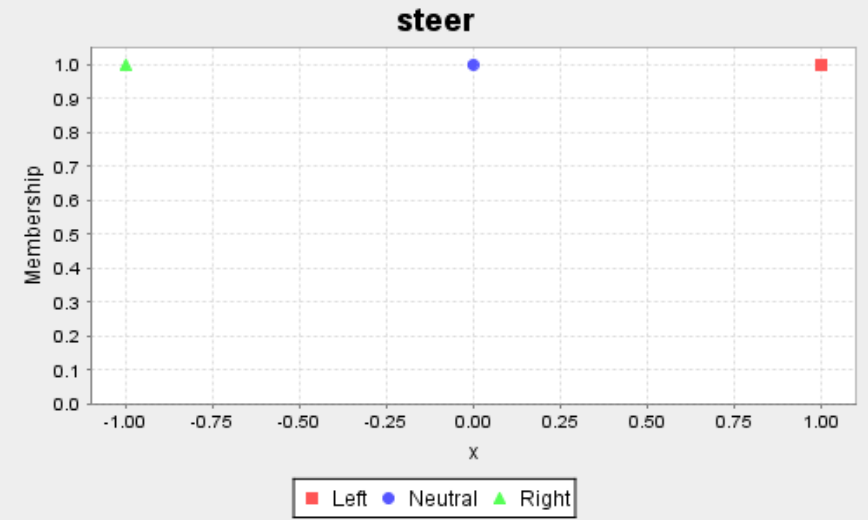

Fig. 8. Three fuzzy sets associated with the desired steering output

function of distance as follows:

$$
\theta(s)=\frac{\left|\operatorname{atan}\left(\frac{\text { waypointIntersectionRadius }}{s}\right)\right|}{2}
$$

where $s$ is the value of the distance variable and waypointIntersectionRadius is a constant.

Let us denote the trapezoidal membership functions as follows:

$\operatorname{trape}(a, b, c, d)(x)=\mu_{\text {trape }}(x, a, b, c, d)=\left\{\begin{array}{cc}0, & x<a, x>d \\ \frac{x-a}{b-a}, & a \leq x \leq b \\ 1, & b<x<c \\ \frac{d-x}{d-c}, & c \leq x \leq d\end{array}\right.$

We defined the membership functions of the contextdependent fuzzy sets of the heading angle variable depending on the distance variable as follows:

- StraightAhead: trape $(-2 \times \theta,-\theta, \theta, 2 \times \theta)$

- SmallLeft: trape $(-5 \times \theta,-3 \times \theta,-2 \times \theta,-\theta)$

- SmallRight: trape $(\theta, 2 \times \theta, 3 \times \theta, 5 \times \theta)$

- LeftDir: trape $(-\pi,-\pi, 5 \times \theta, 0)$

- RightDir: trape $(0,5 \times \theta, \pi, \pi)$

Please refer to Figure 9 for more details.

4) The speed controller: The inputs of the speed controller are distance, speed, heading angle and next angle variables. The output is the desired speed. The controller applies Mamdani's inference. There are ten rules implementing the following strategy:

- The car goes forward most of the time (positive speed). It should only try to reverse in the circumstance that the car is moving at a slow speed away from the target and the distance to the target is not far away.

- Once going backward (negative speed), the car will maintain its direction until one of the following alternatives happens

- The target is in front of the car at a very far distance

- The target is in front of the car at a reasonably far distance and the current backward speed is slow

- The speed of the car is always adjusted to be proportional to the distance to the target. The further the distance, the faster the speed and vice versa. 

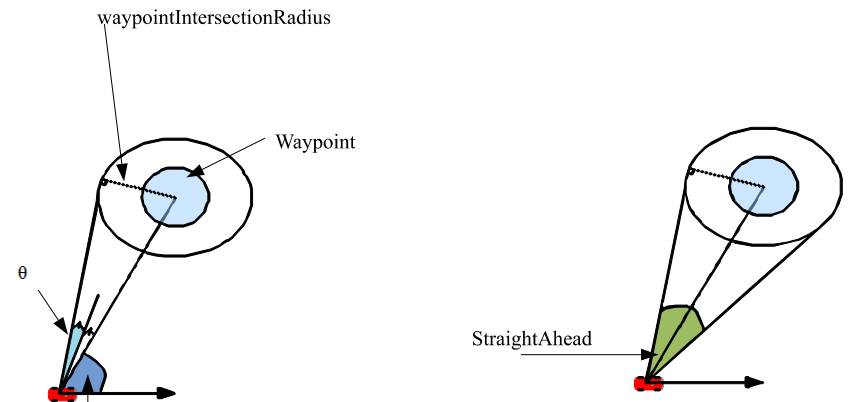

Heading angle



Fig. 9. The meanings of $\theta$ and the fuzzy sets of the heading angle variable
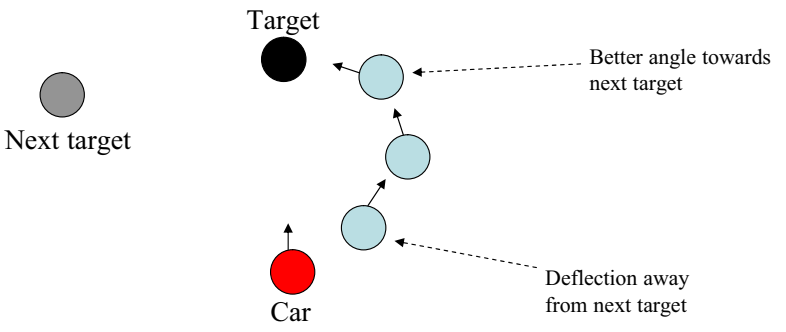

Fig. 10. Steering controller

5) The steering controller: The inputs of the steering controller are distance, heading angle and next angle variables. The output is the desired steering. The controller applies Takagi-Sugeno's fuzzy inference with nine rules. The steering controller always turns the car towards the target until the angle towards the target is within a small tolerance defined by the fuzzy set StraightAhead, in which case, the following rules apply

- If the distance to the target is too near or the car is at a small angle away from the next target, keep neutral steering.

- Otherwise, the car will deflect itself from the next target by a very small angle. See Figure 10 for an example. This will set up the car with a better angle towards the next target in the future when the car turns itself back to the target.

\section{The path planner}

The execution controller described above guarantees to find at least a solution in all situations, i.e find a path that visits both the target and the next target way points. The solution also ensures that the car reaches the target way point in such a way that the next way point can be reached quickly. The speed of the car is reasonably controlled along the path, i.e increase the speed when the distance is far and smoothly slow down on the approach to avoid overshooting. However, the solution given by the execution controller is far from optimal. From observation, it is easily found out that there are situations where the car slows down too much on the approach without realize that it can go quicker if follows the next target directly as the path to the next target will pass through the current target way point. To overcome this problem, we introduce the path planner which basically tries different trajectories to the next target way point directly and takes only the first path that visits the target way point. The path planner is called when the car is at a certain distance close to the target. After the path is computed, all actions of the car to follow that path are stored. Subsequent calls to the controller will return the pre-computed action corresponding to the current state of the car. If any collisions happen, the path planner is called again to compute a new path. The following steps are performed by the path planner:

- Step 1 : Pre-calculate the path to the target

- Step 2 : Store all the car's configurations, i.e position, speed, angle, etc., and the corresponding control commands to follow the path

- Step 3: Take the next configuration of the car on the predicted path and plan a new path to the next target directly. If the current target way point is found on the path, stop the planning and return all the corresponding control commands from the current configuration of the car to when the car hits the target. If it is obvious that the current path will not visit the current target, i.e the car is heading away from the current target, repeat step 3 until a solution is found.

\section{EXPERIMENTS \& RESULTS}

How good a controller is is measured as how many waypoints it can pass within a set time limit of 500 time steps. The number of way points collected is the score of the car after a race. The average score of a controller after 500 races is the final score for that controller. The higher the final score, the better the controller. The competition was organized as two rounds:

- In the first round, each controller was required to race on its own, versus a weak controller and versus an intermediate controller. The weak and intermediate controllers were provided by the organiser for example purposes only. The average of the final scores of these races were used to rank the submitted controllers. Only the top four controllers went to the next round. Table I lists the Competition Score of the final submitted versions of all the controllers in the competition.

- In the final round, the top four controllers competed head-to-head in a round-robin manner. The controller having the most wins was the winner of the competition. Table II lists the names, controller representations and training methods of the top four controllers with highest 
CompetitionScore. Table III lists the solo scores of the top four controllers. And finally, table IV lists the results of running the four controllers with highest Competition Score against each other.

TABLE I

COMPETITIONSCORE

\begin{tabular}{|l|r|}
\hline Controller & CompetitionScore \\
\hline Ho \& Garibaldi & 20.3 \\
Tomoharu & 19.5 \\
Chin Hiong & 19.1 \\
Binatix, Inc. & 19.0 \\
Phil Hingston et al. & 18.7 \\
Bob MacCallum & 16.9 \\
Thomas Haferlach & 16.5 \\
Aravind Gowrisankar & 15.4 \\
Pete Burrow & 15.2 \\
Matt Simmerson & 14.0 \\
\hline Example controllers (Julian Togelius) & 12.6 \\
\hline
\end{tabular}

TABLE II

TOP FOUR COMPETITORS AND THEIR METHODS

\begin{tabular}{|c|c|c|}
\hline Competitors & Methods & Training methods \\
\hline \hline Ho\&Garibaldi & Fuzzy controller & - \\
\hline Tomoharu & Neural networks & Reinforcement learning \\
\hline Chin Hong & Magnetic force field & GA \\
\hline Binatix, Inc. & Feed-forward neural net & Td-learning \\
\hline
\end{tabular}

TABLE III

SOLO SCORE

\begin{tabular}{|l|r|}
\hline Controller & Solo Score \\
\hline Ho \& Garibaldi & 24.4 \\
\hline Binatix, Inc. & 21.03 \\
\hline Chin Hiong & 20.07 \\
\hline Tomoharu & 19.2 \\
\hline
\end{tabular}

TABLE IV

HEAD-TO-HEAD COMPETITION RESULTS

\begin{tabular}{|l|l|l|l|}
\hline & Tomoharu & Chin Hiong & Binatix, Inc \\
\hline Ho\&Garibaldi vs & $17.6 / 11.2$ & $19.2 / 19.1$ & $18.3 / 17.5$ \\
\hline Tomoharu vs & - & $13.0 / 18.8$ & $13.7 / 18.5$ \\
\hline Chin Hiong vs & & - & $19.4 / 19.4$ \\
\hline Binatix, Inc vs & & & - \\
\hline
\end{tabular}

As can be seen, our controller (Ho \& Garibaldi) performed consistently well in both solo racing and in direct competitions. The other controllers in the top four had various performances in different kind of races. Supprisingly, the second top-scoring controller in terms of CompetitionScore, Tomoharu's, was roundly beaten by a large margin by the other finalists in head-to-head racing. After careful examination, it seems that this controller was more specialized towards beating the heuristic controllers used for the scoring function, at the expense of general racing skills. The Binatix, Inc. controller had a very good solo score but has not shown any significant difference in terms of performance compared to the controller of Chin Hong in a direct competition, although the competition between the two has been run 3000 times.

Surprisingly, given the very similar scores, there is a quite a bit of behavioral variation even between the top four controllers. Especially, some controllers rely on stopping and reversing direction when a way point is behind them, whereas others keep a fairly constant speed. Some controllers, e.g the Binatix, Inc and Chin Hong's controllers, drive backwards only. Despite their counter-intuitive driving styles, these controllers are among the best controllers in the competition. The interested readers is particularly referred to [14] for a comprehensive comparison of all the controllers in this competition.

\section{DiscusSiOn \& CONCLUSION}

The focus of the paper is the main controller that actually pilots the car to the target with regard to the next target way point. The main controller does not follow the classical approach of planning the optimal path first and then follow the path as closely as possible, but it was built upon an underlying execution controller that is able to react in realtime based on the current states of the car without referring to any pre-computed path. The execution controller differs from the classical fuzzy controllers in that it utilized the novel idea of context-dependent fuzzy sets [13] instead of the standard type-1 fuzzy sets, thus allowing more flexible fuzzy controllers that can adapt to different context. The purpose of the execution controller is to quickly give a generally good reaction in all situations without performing any expensive high-level reasoning operations, i.e planning. Only in some particular situation where path planning could be quickly performed do we pre-compute the optimal trajectory and follow it as in the classical approach. This approach is a balance between reactivity and accuracy features of the existing approaches. There are also a few additional advantages of this approach

- The number of trainings is dramatically reduced, thus making this approach more suitable to very sophisticated physical model, e.g the real-world model, where the training process is very expensive and timeconsuming. In fact, we do not use any training in this paper at all. However, we believe that by applying reasonable amount of machine learning techniques we could tune-up the membership functions of the fuzzy controllers to achieve better performance.

- The fuzzy approach could avoid some of the unexpected behaviours or counter-intuitive driving style that could be found in evolved controllers. For example, the three best evolved controllers of the CEC 2007 simulated car racing competition all drive backward only.

- The fuzzy model in this paper differs from the classical fuzzy approach in that the membership functions of the variables are not fixed but dependent on some variables. This will hopefully enhance the adaptability of the controllers in more diversified circumstances.

We hope that the techniques used in developing the main controller especially the fuzzy approach that applied in the 
execution controller module, are relevant to more complex car racing simulations as well as to real-world car driving applications. The followings are some ideas/possibilities that should be further investigated:

- Fuzzy approach could be used in combination with machine learning techniques to reduce the number of trainings required and thus making the solution more applicable to the real-world applications. Furthermore, fuzzy approach is particularly useful in a complex environment where the physical model of the environment and the car are partially known or uncertain.

- The classical state-based approach that has been widely used in optimal control consists in computing an optimal trajectory first and then a controller is built to track this trajectory. Typically, the pre-computed path is broken into a numerous critical points which the car should visit in the correct orders. This is very similar to the rules of the point-to-point model used in this paper. It is hoped that the methods/ideas presented in this paper is applicable in a more complex environment such as the Robot Auto-Racing Simulator, The Open Racing Car Simulator (TORCS), etc.

Our research on applying fuzzy techniques in a more complex car racing application like TORCS is on going and will be presented in another paper.

\section{REFERENCES}

[1] DARPA, DARPA Grand Challenge, 2005. [Online]. Available: http://www.grandchallenge.org/
[2] J. Togelius and S. M. Lucas, "Evolving controllers for simulated car racing," in Proceedings of the Congress on Evolutionary Computation, 2005.

[3] — , "Arms races and car races," in Proceeding of Parallel Problem Solving from Nature, 2006.

[4] — "Evolving robust and specialized car racing skills," in Proceedings of the IEEE Congress on Evolutionary Computation, 2006.

[5] S. M. Lucas and J. Togelius, "Point-to-point car racing; evolution versus temporal difference learning," Journal of Information Technology and Intelligent Computing, 2007.

[6] D. Floreano, T. Kato, D. Marocco, and E. Sauser, "Co-evolution of active vision and feature selection," Biological Cybernetics, vol. 90, pp. 218-228, 2004.

[7] B. Chaperot and C. Fyfe, "Improving artificial intelligence in a motocross game," IEEE Symposium on Computational Intelligent and Games, 2006.

[8] P. Abbeel and A. Y. Ng, "Using inaccurate models in reinforcement learning," 2006. [Online]. Available: http://www.cs.stanford.edu/ pabbeel/

[9] R. Coulom, "Reinforcement learning using neural networks, with applications to motor control," Ph.D. dissertation, Institut National Polytechnique de Grenoble, 2002.

[10] M. E. Timin, "The robot auto racing simulator," pp. 109,147, 1995 [Online]. Available: http://rars.sourceforge.net

[11] B. Wymann, TORCS robot tutorial, 2005. [Online]. Available: http://www.berniw.org/

[12] E. Espié and C. G. .et .al, The Open Racing Car Simulator, 1997. [Online]. Available: http://torcs.sourceforge.net/

[13] D. T. Ho and J. M. Garibaldi, "A novel fuzzy inferencing methodology for simulated car racing," in Proceedings of WCCI 2008, 2008.

[14] J. Togelius, S. M. Lucas, D. T. Ho, J. M. Garibaldi, T. Nakashima, C. H. Tan, I. Elhanany, S. Berant, P. Hingston, R. M. MacCallum, T. Haferlach, A. Gowrisankar, and P. Burrow, "The 2007 ieee cec simulated car racing competition," Genetic Programming and Evolvable Machines, 2008. [Online]. Available: http://dx.doi.org/10.1007/s10710-008-9063-0

[15] L. A. Zadeh, "Fuzzy sets," Information and Control, vol. 8, pp. 338$353,1965$.

[16] J. Mendel, Uncertain Rule-Based Fuzzy Logic Systems: Introduction and New Directions. NJ: Prentice-Hall, 2001. 